\title{
Ginsenoside Rg3 protects glucocorticoid-induced muscle atrophy in vitro through improving mitochondrial biogenesis and myotube growth
}

\author{
RYUNI KIM ${ }^{1}$, JEE WON KIM ${ }^{1}$, SANG-JIN LEE ${ }^{2}$ and GYU-UN BAE ${ }^{1}$ \\ ${ }^{1}$ Drug Information Research Institute, College of Pharmacy, Sookmyung Women's University, Seoul 04310; \\ ${ }^{2}$ Research Institute of Aging Related Disease, AniMusCure Inc., Suwon 16419, Republic of Korea
}

Received May 26, 2021; Accepted December 2, 2021

DOI: $10.3892 / \mathrm{mmr} .2022 .12610$

\begin{abstract}
Ginsenoside $\operatorname{Rg} 3$ ( $\operatorname{Rg} 3$ ), amplified by iterative heating processing with fresh ginseng, has a broad range of pharmacological activities and improves mitochondrial biogenesis in skeletal muscle. However, thus far no study has examined how $\operatorname{Rg} 3$ affects myotube growth or muscle atrophy, to the best of the authors' knowledge. The present study was conducted to examine the myogenic effect of $\operatorname{Rg} 3$ on dexamethasone (DEX)-induced myotube atrophy and the underlying molecular mechanisms. Rg3 activated Akt/mammalian target of rapamycin signaling to prevent DEX-induced myotube atrophy thereby stimulating the expression of muscle-specific genes, including myosin heavy chain and myogenin, and suppressing muscle-specific ubiquitin ligases as demonstrated by immunoblotting and immunostaining assays. Furthermore, Rg3 efficiently prevented DEX-triggered mitochondrial dysfunction of myotubes through peroxisome proliferator-activated receptor- $\gamma$ coactivatorl $\alpha$ activities and its mitochondrial biogenetic transcription factors, nuclear respiratory factor-1 and mitochondrial transcription factor A. These were confirmed by immunoblotting, luciferase assays, RT-qPCR and mitochondrial analysis measuring the levels of ROS, ATP and membrane potential. By providing a mechanistic insight into the effect of $\operatorname{Rg} 3$ on myotube atrophy, the present study suggested that $\operatorname{Rg} 3$ has potential as a therapeutic
\end{abstract}

Correspondence to: Professor Gyu-Un Bae, Drug Information Research Institute, College of Pharmacy, Sookmyung Women's University, Cheongpa-ro 47-gil 100, Yongsan, Seoul 04310, Republic of Korea

E-mail: gbae@sookmyung.ac.kr

Dr Sang-Jin Lee, Research Institute of Aging Related Disease, AniMusCure Inc., Seobu-ro 2066, Jang-an, Suwon 16419, Republic of Korea

E-mail: animus_sjlee@animuscure.com

Key words: glucocorticoids, mitochondria, muscle atrophy, myotube growth, $\mathrm{PGC} 1-\alpha$ or nutraceutical remedy to intervene in muscle aging or diseases including cancer cachexia.

\section{Introduction}

Muscle atrophy not only occurs when muscles have certain problems such as denervation or inactivity but also occurs as a systemic response to fasting or various diseases including sepsis, renal and cardiac failure, excessive glucocorticoids (as in Cushing syndrome) and trauma (1). In addition, it also occurs in $80 \%$ of patients with cancer (a condition known as cancer-cachexia) (2). In muscle atrophy, the muscle mass and body weight decrease rapidly, muscles are weakened and disabilities are aggravated because muscle atrophy changes the balance between protein synthesis and degradation pathways toward protein breakdown (3). Consequently, muscle atrophy may deteriorate the quality of life and increase mortality. The degradation of most muscle proteins, including myofibrillar components, are attributable to the ubiquitin-proteasome system and muscle weakening can be explained by the loss of contractile machinery in the event of muscle atrophy $(1,4)$. RING-finger 1 (MuRF1; also known as TRIM63) and atrogin-1 (also known as MAFbx), two E3 ubiquitin ligases specific to muscles, are notably induced by almost all types of muscle atrophy and are upregulated in atrophying muscles by Forkhead box O3 (FoxO3) (5). Akt/mammalian target of rapamycin (mTOR) signaling suppresses FoxO transcription factors that control the expression of the atrogene program thereby increasing net protein accumulation (6). The relevant signaling serves a crucial role in the regulation of muscle protein synthesis and hypertrophy (5). Furthermore, Akt is critical in myoblast differentiation and muscle regeneration (7). However, in the event of fasting or chronic diseases, Akt/mTOR signaling declines leading to the reduction of protein synthesis, while FoxO-dependent expression of the atrogenes increases protein breakdown (1). As the importance of skeletal muscle functions for the maintenance of metabolic health and the quality of life has been accentuated, more attention has been paid to the understanding of molecular regulatory mechanisms of controlling muscle stem cell differentiation and muscle growth and compounds for improvement of muscle functions and mass. 
Panax ginseng C.A. Meyer (P. ginseng) has been used as an herb in northeast Asia for thousands of years (8). In Korean medicine, ginseng has long been used to revitalize the mind and body, prevent aging and increase physical strength and vigor (8). Ginsenosides are the major pharmacologically active compounds of $P$. ginseng and have a variety of biological and physiological activities including anti-inflammatory, anti-diabetic and anti-cancer activities $(9,10)$. Ginsenoside $\mathrm{Rg} 3$ ( $\mathrm{Rg} 3$ ), identified as a protopanaxadiol-type, is amplified by iterative heating processing with fresh and white ginseng. $\operatorname{Rg} 3$ has been found to inhibit the proliferation of breast cancer cells and enhance anti-cancer efficacy compared with other ginsenosides $(9,11) . \operatorname{Rg} 3$ undermines preadipocyte differentiation and the expression of adipogenic markers in 3T3-L1 cells (12). Rg3 enhances mitochondrial functions and the expression of key genes involved in mitochondrial biogenesis thereby improving insulin resistance in skeletal muscles (10). In addition, Rg3 leads to the activation of insulin receptor substrate-1 (IRS-1) without altering the classical AMP-activated protein kinase (AMPK) pathway and upregulates IRS-1, Akt and Glut4 in L6 myotubes thereby improving insulin signaling and glucose uptake (13). Recent advances in muscle biology, particularly in relation to anabolic and catabolic stimulation for molecular regulation of muscle mass and function, have spurred renewed interest in pharmacological treatments to prevent muscle wasting (14). The authors of the present study have previously reported the effect of BST204 (ginseng extract) or Rg3 on muscle atrophy induced by TNF- $\alpha$, which is known to be secreted in the inflammatory response $(15,16)$. However, there are no studies examining the effect of ginsenoside $\mathrm{Rg} 3$ on corticosteroid drug-induced myotube atrophy, to the best of the authors' knowledge.

The present study investigated the myogenic effect and the underlying molecular mechanisms of ginsenoside $\mathrm{Rg} 3$ on dexamethasone (DEX)-induced myotube atrophy. It was found that Rg3 activated Akt/mTOR signaling in DEX-induced myotubes thereby suppressing muscle atrophy-related signaling and improved peroxisome proliferator-activated receptor $\gamma$ coactivator 1- $\alpha$ (PGC1- $\alpha)$-mediated mitochondrial functions thereby preventing myotube atrophy. The present study demonstrated the effect of $\operatorname{Rg} 3$ on myotube growth and PGC1- $\alpha$-mediated mitochondrial functions in atrophic myotubes. Its findings provided important clues to our understanding of how $\mathrm{Rg} 3$ protects DEX-induced myotube atrophy in molecular level, suggesting that $\mathrm{Rg} 3$ might be a potential therapeutic to intervene muscle aging or diseases including cancer cachexia.

\section{Materials and methods}

Reagents. Rg3 (purity >98.0\%, ASB-00007217-025) was purchased from ChromaDex. Serums including Fetal bovine serum (FBS), horse serum (HS) and Dulbecco modified Eagle's medium (DMEM) were purchased from Thermo Fisher Scientific, Inc. MitoTracker Red CM-H2XRos (cat. no. M-7512), ATP Determination kit (cat. no. A-22066) and JC-1 (5,5,6,6-Tetrachloro-1,1,3,3-tetraethylbenzimid azolylcarbocyanine iodide; cat. no. T-3168) were bought from Molecular Probes (Thermo Fisher Scientific, Inc.). Dexamethasone (DEX; cat. no. D4902) and all other chemicals were from Sigma-Aldrich (Merck KGaA).
Cell culture. Myoblast $\mathrm{C} 2 \mathrm{C} 12$ cells were cultured at $37^{\circ} \mathrm{C}$ with $5 \% \mathrm{CO}_{2}$ as described previously (7). $\mathrm{C} 2 \mathrm{C} 12$ cells were incubated for $48 \mathrm{~h}$ after thawing. During incubation, the cells were detached by Trypsin/EDTA (cat. no. LS015-08; Welgene) to avoid contact between cells. C2C12 cells were tested for mycoplasma by PCR within two weeks after thawing. No mycoplasma contamination was detected. For the DEX-induced atrophy study, the differentiation of $\mathrm{C} 2 \mathrm{C} 12$ cells was induced in DMEM containing 2\% HS (differentiation medium, DM) at $37^{\circ} \mathrm{C}$ with $5 \% \mathrm{CO}_{2}$ for three days and the resultant cells were treated with $10 \mu \mathrm{M} \mathrm{DEX}$ at $37^{\circ} \mathrm{C}$ with $5 \% \mathrm{CO}_{2}$ for 1 day. Thereafter, the concentration of ginsenoside $\mathrm{Rg} 3$ was indicated and the cells were incubated in DM for an additional day (D4) (17).

Immunoblotting analysis, immunocytochemistry and microscopy. Immunoblotting analysis was carried out as previously described $(7,18)$. Briefly, C2C12 cells were lysed in extraction buffer (50 mM Tris- $\mathrm{HCl}, \mathrm{pH} 7.4,150 \mathrm{mM} \mathrm{NaCl}, 1.2 \mathrm{mM} \mathrm{MgCl}_{2}$, $1 \mathrm{mM}$ EGTA, $1 \%$ Triton X-100, $10 \mathrm{mMNaF}$ and $\left.1 \mathrm{mMNa}_{3} \mathrm{VO}_{4}\right)$ containing complete protease inhibitor cocktail (Roche Diagnostics) followed by a Bradford assay (cat. no. 50000006; Bio-Rad Laboratories, Inc.) for protein quantification. Total protein $(30 \mu \mathrm{g} /$ lane) was separated via SDS-PAGE on $6 \%$ gel for MHC, pan-Cadherin, p-mTOR, mTOR and a $10 \%$ gel for the rest. Subsequently, protein was transferred onto PVDF membranes (cat. no. IPVH00010; MilliporeSigma). The membranes were blocked with $5 \%$ skim milk for $30 \mathrm{~min}$ at room temperature, followed by overnight incubation with primary antibodies at $4{ }^{\circ} \mathrm{C}$. After washing three times with TBS with $0.1 \%$ Tween-20 for $5 \mathrm{~min}$ at room temperature, the membranes were incubated with a HPR-conjugated secondary antibodies (1:5,000; cat. nos. 7076S and 7064S; Cell Signaling Technology, Inc.) for $1 \mathrm{~h}$ at room temperature. Protein bands were visualized using an ECL Kit (cat. no. ABC-3001; AbClon, Inc.) and semi-quantified using ImageJ version 2.1.4.7 (National Institutes of Health).

In this study, the following primary antibodies were used: myosin heavy chain (MHC; 1:500; MF-20; Developmental Studies Hybridoma Bank), phosphorylated (p)-Akt (1:1,000; cat. no. 4060), Akt (1:1,000; cat. no. 9272), p-mTOR (1:1,000; cat. no. 5536), mTOR (1:1,000; cat. no. 2983), p-p70S6K (1:1,000; cat. no. 9234), p70S6K (1:1,000; cat. no. 2708), PGC1- $\alpha$ (1:500; cat. no. 2178), nuclear respiratory factor 1 (NRF1; 1:1,000; cat. no. 69432), mitochondrial transcription factor A (Tfam; 1:1,000; cat. no. 8076) (Cell Signaling Technology, Inc.), myogenin (1:1,000; sc-52903), $\alpha$-Tubulin (1:10,000; cat. no. sc-5286, Santa Cruz Biotechnology, Inc.), MuRF1 (1:500; cat. no. GTX110475, GeneTex, Inc.), atrogin-1 (1:500; cat. no. AP2041, ECM Biosciences) and pan-Cadherin (1:10,000; cat. no. C3678; Sigma-Aldrich; Merck KGaA). Pan-Cadherin, Akt, mTOR, p70S6K and $\alpha$-Tubulin were blotted as reference proteins.

Immunostaining was carried out for $\mathrm{MHC}$ expression as previously described (18). Briefly, the differentiated C2C12 myotubes were fixed with $4 \%$ paraformaldehyde for $10 \mathrm{~min}$ at room temperature and permeabilized with $0.5 \%$ Triton-X 100 for $10 \mathrm{~min}$ at room temperature. They were then blocked with 5\% goat serum (cat. no. 16210064; Gibco; Thermo Fisher Scientific, Inc.) for $30 \mathrm{~min}$ at room temperature followed by 
immunostaining using MHC antibodies (1:200) for $1 \mathrm{~h}$ at room temperature and Alexa 568-conjugated secondary antibodies (1:200) (Molecular Probes; Thermo Fisher Scientific, Inc.) for $1 \mathrm{~h}$ at room temperature. The images were visualized by fluorescence microscopy in five random fields using a Zeiss microscope (magnification, x200; Axio Observer Z1; Zeiss AG) and the diameter of the myotubes was measured using ImageJ (version 2.1.4.7; National Institutes of Health). MHC-positive myotube diameters were measured transversely with 15 myotubes in one well of 6 wells culture plate.

RNA extraction and reverse transcription-quantitative (RT-q) $P C R$. Total RNA was extracted from the cells as previously described (19). In short, total RNA was extracted from cells at $100 \%$ confluency in a $60-\mathrm{mm}$ culture plate using an easy-spin Total RNA Extraction kit (cat. no. 17221; Intron Biotechnology, Inc.) according to the manufacturer's instructions. Purity and concentration of RNA were measure using a spectrophotometer (Epoch, BioTek Instruments, Inc.; Agilent Technologies, Inc.), followed by cDNA synthesis using an iScript cDNA synthesis kit (cat. no. 1708890; Bio-Rad Laboratories, Inc.) according to the manufacturer's instructions.

RT-qPCR analysis was carried out as previously described according to the manufacturer's instructions (19). The qPCR reaction was performed using SYBR Green PCR Master Mix (cat. no. 4309155; Thermo Fisher Scientific, Inc.) and QuantStudio3 (Applied Biosystems; Thermo Fisher Scientific, Inc.). The reaction conditions were as follows: Initial denaturation at $95^{\circ} \mathrm{C}$ for $30 \mathrm{sec}$; followed by $95^{\circ} \mathrm{C}$ for $5 \mathrm{sec}$ and $60^{\circ} \mathrm{C}$ for $30 \mathrm{sec}(40$ cycles). One experiment was performed with three technical replicates. All the data were normalized to the expression of housekeeping gene, $18 \mathrm{~S}$ ribosomal RNA. The Livak-Schmittgen $2^{-\Delta \Delta \mathrm{Cq}}$ method was used to analyze the relative gene expression data (20). The primer sequences used in this study were: PGC1- $\alpha, 5$ '-ATCTACTGC CTGGGGACCTT-3', 5'-ATGTGTCGCCTTCTTGCTCT-3'; NRF1, 5'-GCCGTCGGAGCACTTACT-3', 5'-CTGTTCCAA TGTCACCACC-3'; Tfam, 5'-CACCCAGATGCAAAACTT TCAG-3', 5'-CTGCTCTTTATACTTGCTCACAG-3'; and 18S rRNA, 5'-AGGGGAGAGCGGGTAAGAGA-3', 5'-GGA CAGGACTAGGCGGAACA-3'.

Detection of mitochondrial reactive oxygen species (ROS), mitochondrial membrane potential (MMP) and ATP content. The mitochondrial ROS generation was determined using MitoTracker Red CM-H2XRos. C2C12 myotubes were incubated for $30 \mathrm{~min}$ at $37^{\circ} \mathrm{C}$ using $100 \mathrm{nM}$ MitoTracker Red. Following the incubation, the resultant cells were washed and lysed with $0.5 \%$ Triton X-100. The cell lysates were centrifuged at $4^{\circ} \mathrm{C}$ at $1,500 \mathrm{x} g$ for $10 \mathrm{~min}$. The resultant supernatants were measured using a fluorescence spectrometer (GloMax Discovery; Promega Corporation) at an excitation wavelength of $515 \mathrm{~nm}$ and an emission wavelength of $535 \mathrm{~nm}$. The optical density values were normalized to protein levels (21). Fluorescence assay was carried out using JC-1 to determine MMP. In short, C2C12 myotubes were cultured in a 96-well plate under different treatment conditions, incubated for $30 \mathrm{~min}$ at $37^{\circ} \mathrm{C}$ using $10 \mu \mathrm{g} / \mathrm{ml}$ of JC- 1 and analyzed thereafter using a fluorescence spectrometer (GloMax Discovery; Promega Corporation). The quantitative analysis was conducted using the fluorescence ration (590 to $530 \mathrm{~nm}$ ) (22). The ATP Determination kit (Molecular Probes; Thermo Fisher Scientific, Inc.) was used to measure the total ATP content, as instructed by the manufacturer.

Peroxisome proliferator-activated receptor- $\gamma$ coactivatorl $\alpha$ (PGC1- $\alpha$ ) luciferase assay. PGC1- $\alpha$ luciferase assay was carried out as previously described (23). The 5' flanking sequence of the mouse PGC1- $\alpha$ gene was amplified by PCR and subcloned into the pGL3 basic reporter gene vector. In relation to the transcriptional start site, the plasmid containing the regions ranging from +78 and $-2,533$ is referred to as a $2 \mathrm{~kb}$ promoter. $\mathrm{C} 2 \mathrm{C} 12$ cells were transfected with reverse transfection method. In short, $\mathrm{C} 2 \mathrm{C} 12$ were seeded in a 12 -well plate at a density of $1.5 \times 10^{5}$ cells per well. The cells were then transfected with $1 \mu \mathrm{g}$ of a luciferase plasmid encoding the full $2 \mathrm{~kb}$ promoter region of PGC1- $\alpha$ (pPGC1- $\alpha$-Luc; Addgene, Inc.) and $300 \mathrm{ng}$ of $\beta$-galactosidase using Lipofectamine ${ }^{\circledR} 2000$ (Invitrogen; Thermo Fisher Scientific, Inc.). After 24 h, cells were transferred into DM and maintained for $48 \mathrm{~h}$ to differentiate at $37^{\circ} \mathrm{C}$ with $5 \% \mathrm{CO}_{2}$. Thereafter, cells were harvested and luciferase activity was determined using luminometer with Luciferase Reporter Assay System (cat. no. E1500, Promega Corporation). The results were normalized to the co-transfected $\beta$-galactosidase enzyme activity measured by reaction with ONPG (cat. no. 73660; Sigma-Aldrich; Merck KGaA), a substrate of $\beta$-galactosidase. Experiments were carried out in triplicates and repeated at least three times independently.

Statistical analysis. PGC1- $\alpha$ luciferase assay and RT-qPCR were performed in three technical replicates and the data was expressed as means \pm SEM. Immunoblotting and immunostaining were performed in three independent experiments and the data was expressed as means \pm SD. One-way ANOVA analysis followed by Bonferroni's post hoc test was used. In DEX-induced atrophy experiments, the DEX/Rg3-treated group was compared with DEX-treated group to access the significance of the difference. $\mathrm{P}<0.05$ was considered to indicate a statistically significant difference.

\section{Results and Discussion}

Treatment of Rg3 with DEX suppresses muscle atrophy-related signaling. Glucocorticoids facilitate protein degradation while inhibiting protein synthesis in skeletal muscles and function as an important factor for the development of muscle atrophy under various catabolic conditions (24). DEX, a synthetic glucocorticoid, has been used for a simple in vitro and in vivo model of muscle atrophy $(17,25)$. Therefore, whether Rg3 can protect myotubes from DEX-induced muscle atrophy was investigated. The differentiation of $\mathrm{C} 2 \mathrm{C} 12$ myoblasts was induced to differentiate for three days and the resultant C2C12 myoblasts were treated with $10 \mu \mathrm{M}$ DEX with vehicle (DMSO) or Rg3 for one day thereafter (D4). The relevant cells were subjected to immunoblotting analysis to discover muscle specific proteins (MHC and myogenin) and muscle specific ubiquitin ligases (atrogin-1 and MuRF1). As shown in Fig. $1 \mathrm{~A}$ and $\mathrm{B}$, treatment of DEX decreased the formation of myotubes diameters, indicating muscle atrophy. While treatment with Rg3 increased the diameters of DEX-treated 
A $\mathrm{C} 2 \mathrm{C} 12(\mathrm{D} 4)$
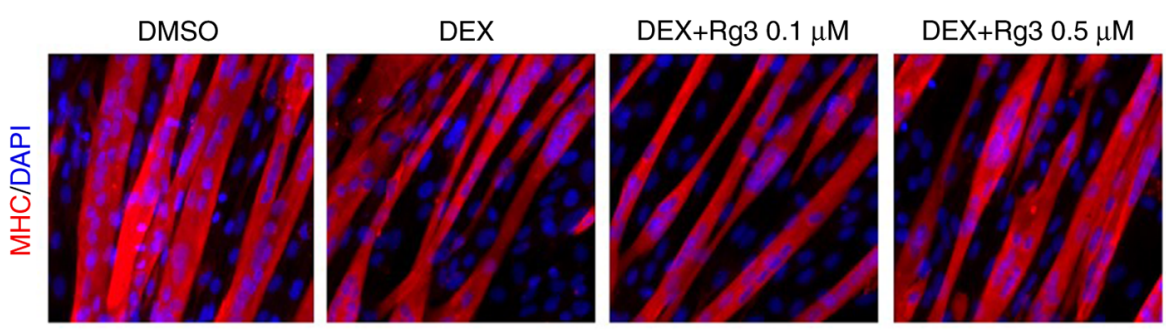

$\mathrm{DEX}+\mathrm{Rg} 31 \mu \mathrm{M}$
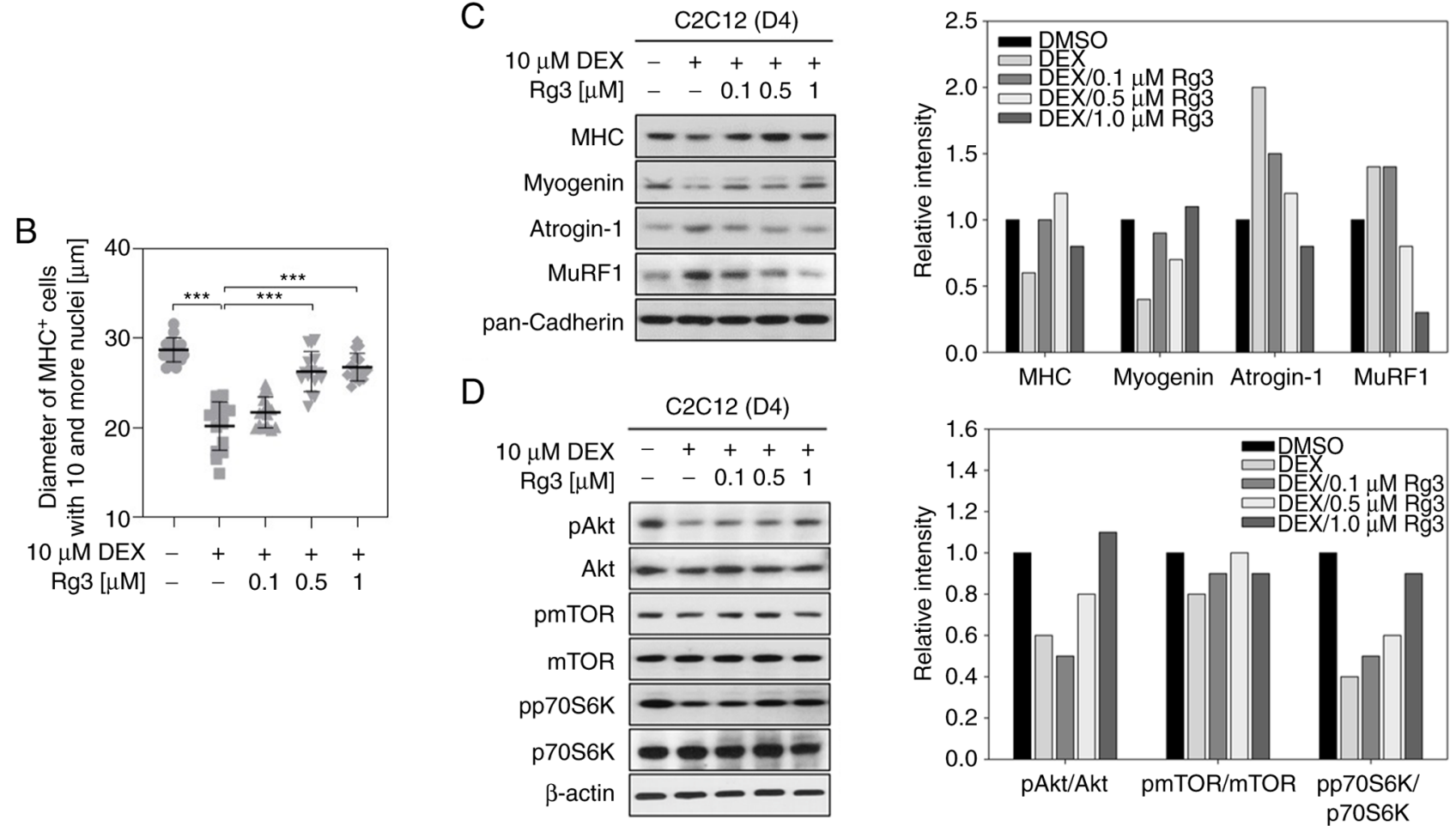

Figure 1. Rg3 treatment with DEX attenuates atrophy-related signaling in myotubes. (A) To prepare myotubes, C2C12 myoblasts were differentiated in DM for three days and the resultant myotubes were treated with DEX with vehicle or the indicated concentration of Rg3 in fresh DM for an additional day (D4). MHC immunostaining was carried out to analyze the myotube formation and DAPI was used to visualize nuclei. (B) Quantification of myotube diameter from data shown in (A). All values represent means $\pm \mathrm{SD}$. Control and Rg3/DEX-treated group were compared with DEX-treated group, ${ }^{* * *} \mathrm{P}<0.002 ;$ one-way ANOVA with post Bonferroni's multiple comparison test. (C) The resultant myotube lysates were subjected to immunoblotting analysis. The signal intensity of MHC, myogenin, atrogin-1 and MuRF1 were quantified and the relative values were normalized to pan-Cadherin. (D) Total and phosphorylated forms of Akt, mTOR and p70S6K from (A) were analyzed by immunoblotting. The signal intensity of p-Akt, p-mTOR and p-p70S6K was quantified and the relative values were normalized to Akt, mTOR and p70S6K, respectively. Rg3, ginsenoside Rg3; DEX, dexamethasone; DM, differentiation medium; MHC, myosin heavy chain; MuRF1, RING-finger 1; p-, phosphorylated.

myotubes in dose-dependent manner. Immunoblotting showed that the expression of MHC and myogenin were decreased in the DEX-treated cells. The treatment with $\mathrm{Rg} 3$ reversed the decrease of these proteins in DEX-treated myotubes. DEX treatment enhanced the levels of expression of atrogin-1 and MuRF1 in vehicle-treated myotubes. By contrast, the treatment with $\operatorname{Rg} 3$ reduced the expression of the same ligases dose-dependently in DEX-treated myotubes, similar to those in control myotubes, from the concentration of $1 \mu \mathrm{M} \mathrm{Rg} 3$ (Fig. 1C). The molecular regulatory mechanism of $\operatorname{Rg} 3$ in DEX-induced myotubes was also investigated. DEX-treated myotubes displayed decreased phosphorylation of Akt and its downstream effectors, mTOR and p70S6K (Fig. 1D). When treated with $\mathrm{Rg} 3$, the phosphorylated levels of Akt, mTOR and p70S6K were dose-dependently restored in DEX-treated myotubes, similarly to those in vehicle-treated myotubes
(Fig. 1D). The signal intensities were quantified and the relative values were normalized to pan-Cadherin, Akt, mTOR and p70S6K, respectively (Fig. 1C and D). The appropriate dose was analyzed in Fig. 1 and a follow-up experiment was conducted with a dose of $1 \mu \mathrm{M}$. Muscle protein breakdown is induced by glucocorticoid primarily due to ubiquitin-proteasome-dependent proteolysis (24). The signaling pathway stimulated by glucocorticoids involved a decrease in protein synthesis and an upregulation of atrogenes, partially by reducing PI3K/Akt signaling while increasing FoxO activity (6), indicating that Rg3 suppresses the effect of DEX in myotube atrophy by activating Akt/mTOR signaling.

Rg3 improves myotube growth through activation of Akt/mTOR signaling in the presence of DEX. To examine the effect of $\operatorname{Rg} 3$ on myotube growth in response to DEX, the differentiation of 
A

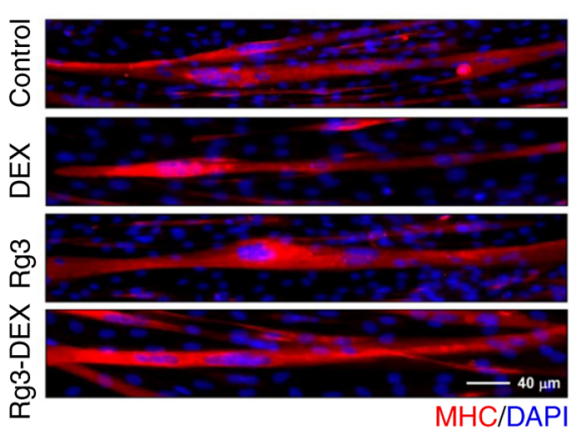

$\mathrm{B}$

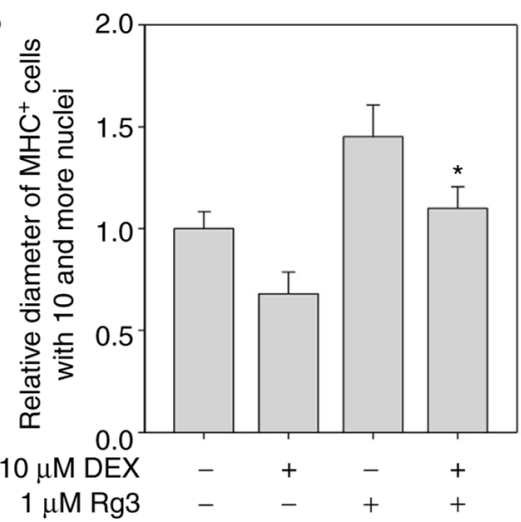

C

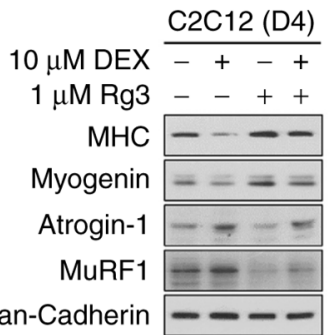

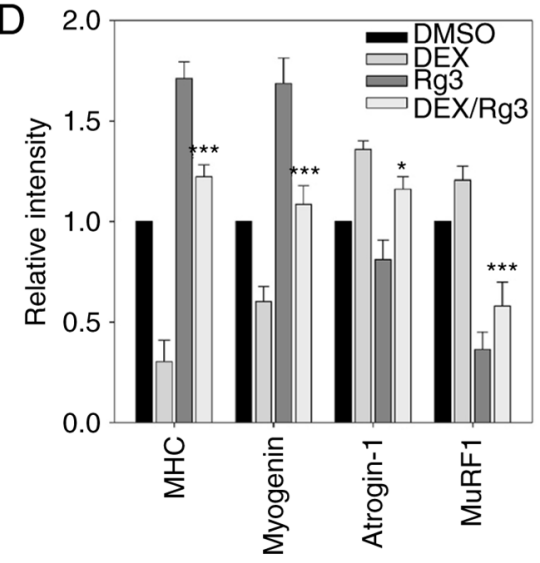

$\mathrm{E}$

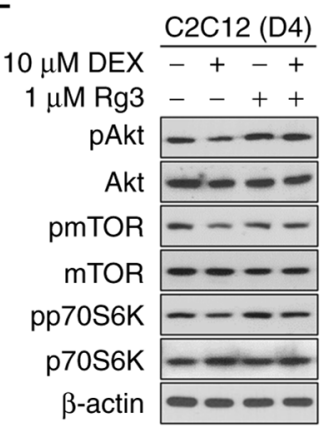

$\mathrm{F}$

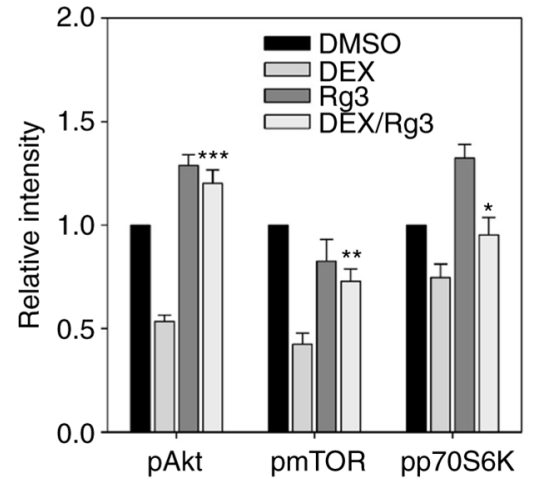

Figure 2. Rg3 activates Akt/mTOR signaling in the presence of DEX thereby promoting myotube growth. (A) C2C12 myoblasts were differentiated in DM for three days to make myotubes and the resultant myotubes were treated with DEX with vehicle or Rg3 for one day (D4). MHC immunostaining was carried out to analyze the myotube formation. DAPI was used to visualize nuclei. (B) Quantification of average myotube diameter from data shown in (A). All values represent means \pm SD. Rg3/DEX-treated group was compared with DEX-treated group, ${ }^{*} \mathrm{P}<0.033$; one-way ANOVA with post Bonferroni's multiple comparison test. (C) $\mathrm{C} 2 \mathrm{C} 12$ myotubes from similar experiments shown in (A) were subjected to immunoblotting analysis. (D) Quantification of blots from three independent experiments similarly performed as shown in (C). The signal intensity of MHC, myogenin, atrogin-1 and MuRF1 was quantified and the relative values were normalized to pan-Cadherin. All values represent means \pm SD. Rg3/DEX-treated group was compared with DEX-treated group, ${ }^{*} \mathrm{P}<0.033$, ${ }^{* * *} \mathrm{P}<0.001$; one-way ANOVA with post Bonferroni's multiple comparison test. (E) C2C12 myotubes from similar experiments shown in (A) were subjected to immunoblotting analysis. (F) Quantification of blots from three experiments similarly performed as shown in (E). The signal intensity of p-Akt, p-mTOR and p-p70S6K was quantified and the relative values were normalized to Akt, mTOR and p70S6K, respectively. All values represent means \pm SD. Rg3/DEX-treated group was compared with DEX-treated group, ${ }^{*} \mathrm{P}<0.033,{ }^{* *} \mathrm{P}<0.002,{ }^{* * *} \mathrm{P}<0.001$; one-way ANOVA with post Bonferroni's multiple comparison test. Rg3, ginsenoside Rg3; DEX, dexamethasone; DM, differentiation medium; MHC, myosin heavy chain; MuRF1, RING-finger 1; p-, phosphorylated.

$\mathrm{C} 2 \mathrm{C} 12$ myoblasts was induced for three days and the resultant C2C12 myoblasts were treated thereafter with DEX together with or without $\mathrm{Rg} 3$ for one day (D4). Thereafter, the resultant myotubes were subjected to immunostaining for MHC to retrieve myotube formation. $\mathrm{Rg} 3$ treatment increased the formation of large multinucleated myotubes compared with vehicle treatment (Fig. 2A). As expected, myotube formation was blocked by DEX-treatment but Rg3 treatment recovered myotube formation in DEX-treated cells leading to the presence of larger multinucleated myotubes. Based on the quantification of myotube diameters, DEX treatment led to decreases in myotube diameter and it was partially blocked by Rg3, similar to the level of vehicle-treated control (Fig. 2B). Countermeasures developed to block or attenuate the muscle mass debilitating process can provide major therapeutic benefits for a variety of clinical conditions. During muscle atrophy, atrogin-1 breaks down proteins that promote protein synthesis, while MuRF1 degrades several components of the sarcomeric thick filaments including MHC and the cytoskeleton through the ubiquitylation $(26,27)$. Thus, by blocking MuRF1 activation, Akt/mTOR signaling prevents the breakdown of the thick filament in the event of muscle atrophy (27).

Replica experiments were conducted to determine the levels of expression of muscle-specific proteins and E3 ubiquitin ligases. As shown in Fig. $2 \mathrm{C}$ and $\mathrm{D}, \mathrm{Rg} 3$ treatment increased MHC and myogenin levels, while DEX treatment decreased these proteins. Consistently with the immunoblotting data shown in Fig. 1A, the levels of expression of MHC and myogenin were restored as the effect of $\operatorname{Rg} 3$ treatment on atrophic myotubes, similar to that on vehicle control. $\operatorname{Rg} 3$ treatment reduced the expression of atrogin-1 and MuRF1 in DEX-treated myotubes, compared with the DEX-treated control (Fig. 2C and D). Furthermore, Rg3 treatment markedly increased the levels of phosphorylated Akt and its downstream substrates, mTOR and p70S6K (Fig. 2E and F). The levels of active Akt, mTOR and p70S6K decreased in DEX-treated myotubes and $\mathrm{Rg} 3$ treatment partially restored the levels of phosphorylation of the relevant proteins in DEX-treated 
A

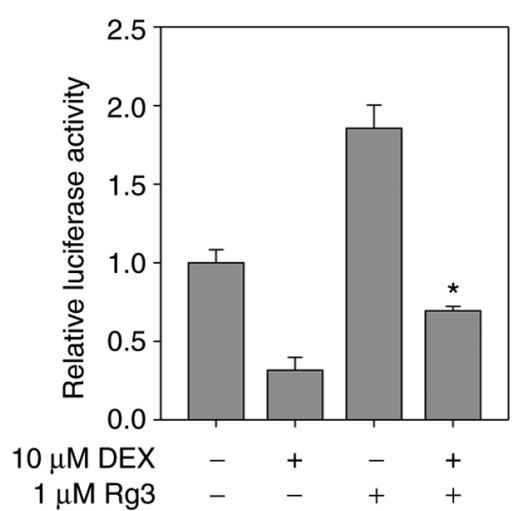

D

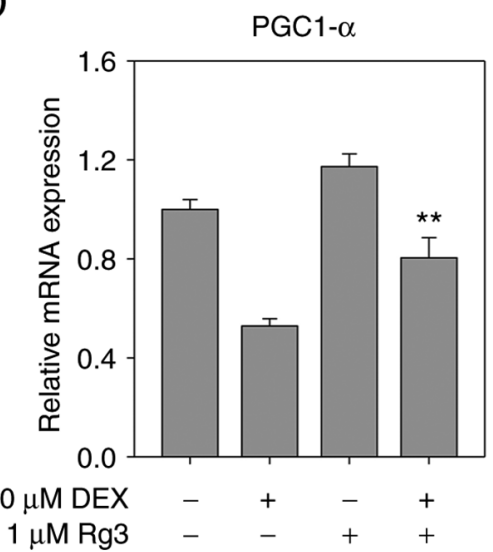

B

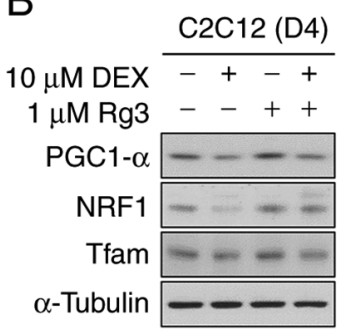

C
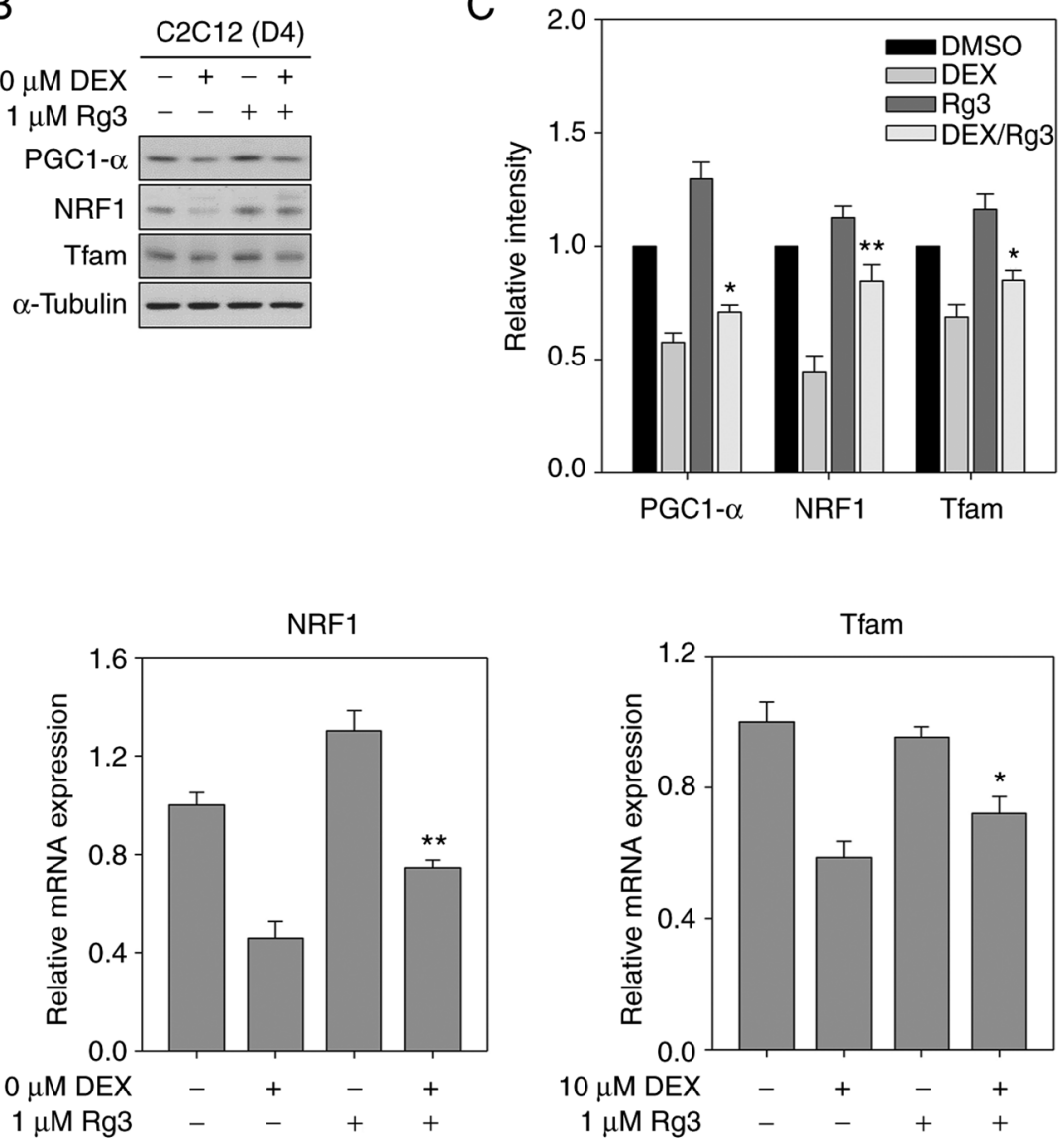

Figure 3. Rg3 promotes the activity of PGC1- $\alpha$ in DEX-induced atrophic myotubes. (A) C2C12 myoblasts were transiently transfected with a PGC1- $\alpha$-responsive luciferase reporter gene and, $24 \mathrm{~h}$ after transfection, cells were transferred into DM without any other substances. DEX with vehicle or Rg3 were treated with fresh DM and maintained additionally for one day. Luciferase assay was carried out thereafter. Experiments were conducted in three technical replications and data were presented as the means \pm SEM. Rg3/DEX-treated group was compared with DEX-treated group, ${ }^{*} \mathrm{P}<0.033$; one-way ANOVA with post Bonferroni's multiple comparison test. (B) $\mathrm{C} 2 \mathrm{C} 12$ myoblasts were differentiated in DM for three days to prepare myotubes and the resultant myotubes were treated with DEX with vehicle or Rg3 for one day (D4). Thereafter, immunoblotting analysis was conducted with the resultant myotubes. (C) Quantification of three independent experiments performed as shown in (B). The intensity of PGC1- $\alpha$, NRF1 and Tfam was quantified and the relative values were normalized to $\alpha$-Tubulin. Data from three independent experiments were presented as the means $\pm 1 \mathrm{SD}$. Rg3/DEX-treated group was compared with DEX-treated group, ${ }^{*} \mathrm{P}<0.033,{ }^{* *} \mathrm{P}<0.002$; one-way ANOVA with post Bonferroni's multiple comparison test. (D) RT-qPCR analysis for PGC1- $\alpha$, NRF1 and Tfam in C2C12 myoblasts from similar experiments shown in (B). All values from control sample were set to 1.0. Experiments were performed in three technical replications and data presented as the means \pm SEM. Rg3/DEX-treated group was compared with DEX-treated group, ${ }^{*} \mathrm{P}<0.033$, ${ }^{* *} \mathrm{P}<0.002$; one-way ANOVA with post Bonferroni's multiple comparison test. Rg3, ginsenoside Rg3; PGC1- $\alpha$, peroxisome proliferator-activated receptor $\gamma$ coactivator 1- $\alpha$; DEX, dexamethasone; NRF1, nuclear respiratory factor 1; Tfam, mitochondrial transcription factor A.

myotubes, similarly to those in the control myotubes (Fig. 2E and F). Sustained protein synthesis promotes muscle hypertrophy, thereby overcoming muscle atrophy (28). Akt is critical in myogenic differentiation because it induces MyoD heterodimerization with E proteins leading to the expression of muscle-specific proteins including MHC and myogenin (7). Upregulation of Akt/mTOR signaling stimulates protein synthesis leading to muscle growth and hypertrophy and Akt activation phosphorylates main mediators of skeletal muscle atrophy (including E3 ubiquitin ligases, MuRF1 and atrogin-1) thereby blocking the transcriptional upregulation of them, consequently inhibiting the nuclear translocation of the FoxO transcriptional factors (29). In addition, Ginsenoside Rg1 has been reported to promote myoblast differentiation and prevent DEX-induced myotube atrophy though activation of $\mathrm{Akt}$ signaling (30). Rb1 and Rb2 activates Akt/mTOR signaling and promotes the heterodimerization of MyoD and E proteins, thereby inducing skeletal muscle hypertrophy (31). Given these results, it can be seen that $\mathrm{Rg} 3$ inhibits muscle-specific E3 ubiquitin ligases mediated by activation of Akt/mTOR signaling thereby preventing myotube atrophy induced by DEX.

Rg3 upregulates the activity and expression of PGC1- $\alpha$ in DEX-induced myotubes. PGC1- $\alpha$ is a master regulator of mitochondrial biogenesis through gene expression and post-translational modification (32). Whether $\mathrm{Rg} 3$ modulates the activity of PGC1- $\alpha$ in DEX-treated myotubes was examined. C2C12 myoblasts were transiently transfected with a PGC1- $\alpha$-responsive luciferase reporter and, after $24 \mathrm{~h}$, the differentiation of myoblasts was induced in the presence of DEX with vehicle or $\operatorname{Rg} 3$ for one day (D2) and luciferase assay was carried out thereafter. According to the result, $\mathrm{Rg} 3$ treatment increased the PGC1- $\alpha$-luciferase activity dose-dependently (Fig. 3A). The treatment with DEX markedly reduced PGC1- $\alpha$-luciferase activity, which was partially 
A

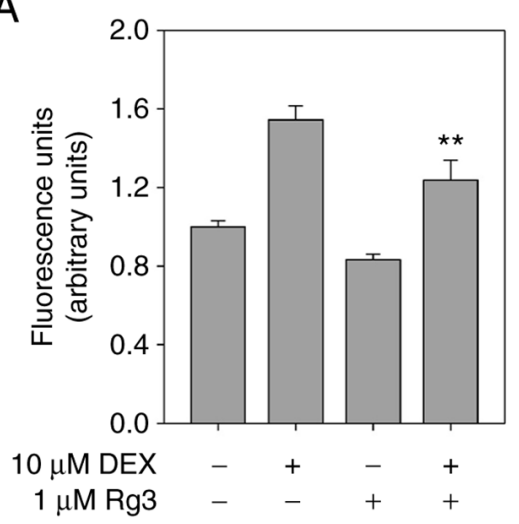

B

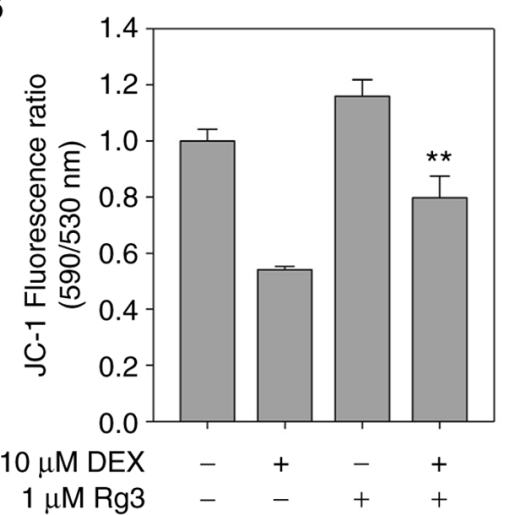

C

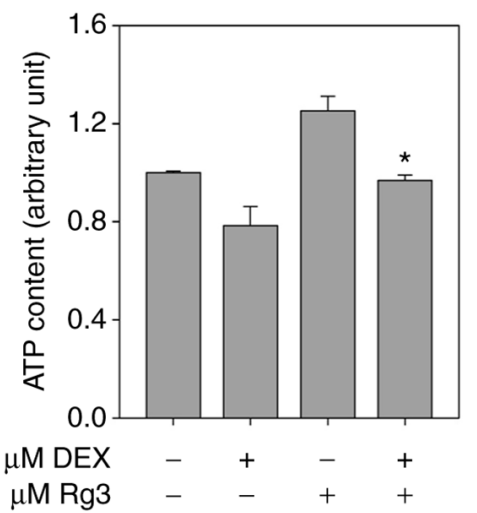

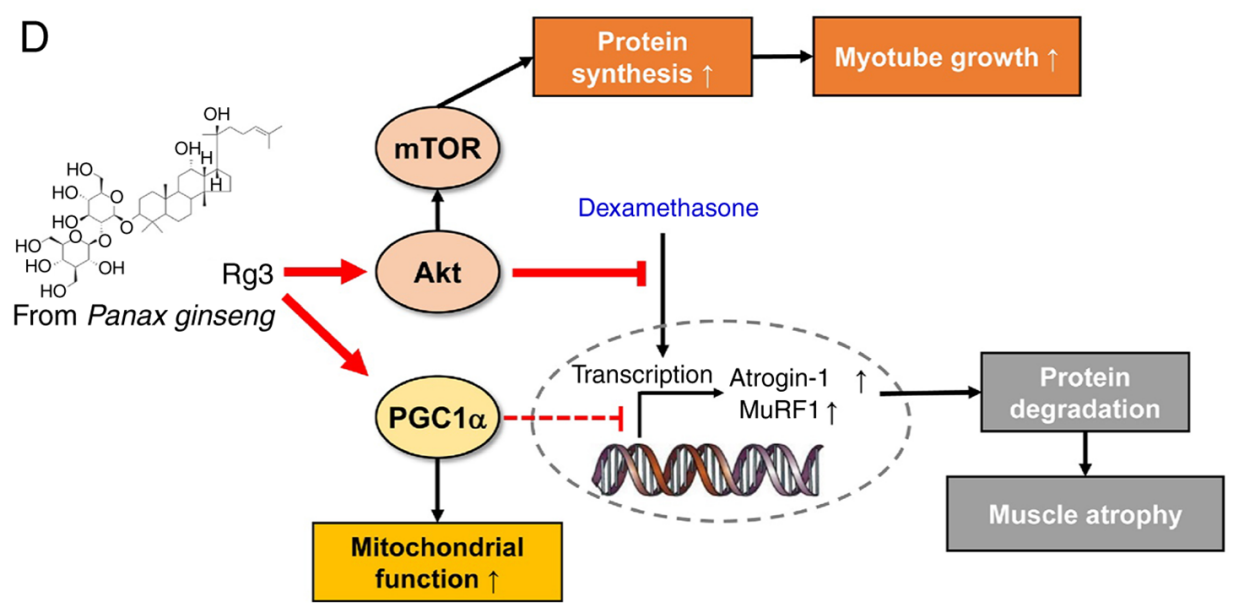

Figure 4. Rg3 suppresses the production of mitochondrial ROS induced by DEX. C2C12 myotubes were treated with DEX with vehicle or Rg3 for additional one day (D4). (A) Mitochondrial ROS production analysis. Fluorescence intensity is given as arbitrary units and values presented as means \pm SD $(\mathrm{n}=5)$. Rg3/DEX-treated group was compared with DEX-treated group, ${ }^{* *} \mathrm{P}<0.002$; one-way ANOVA with post Bonferroni's multiple comparison test. (B) Mitochondrial membrane potential analysis. Fluorescence ratio (590 to $530 \mathrm{~nm}$ ) was used for quantitative analysis. Values presented are as means \pm SD $(\mathrm{n}=4)$. Rg3/DEX-treated group was compared with DEX-treated group, ${ }^{* *} \mathrm{P}<0.002$; one-way ANOVA with post Bonferroni's multiple comparison test. (C) The determination of ATP content. The total ATP content was measured using ATP determination kit. Values presented are as means \pm SD ( $=4$ ). Rg3/DEX-treated group was compared with DEX-treated group, ${ }^{*} \mathrm{P}<0.033$; one-way ANOVA with post Bonferroni's multiple comparison test. (D) Model of the effect and molecular mechanisms of ginsenoside $\operatorname{Rg} 3$ on DEX-induced myotube atrophy. $\operatorname{Rg} 3$ protects DEX-induced myotube atrophy through the activation of Akt/mTOR signaling and suppresses muscle-specific ubiquitin ligases. In addition, Rg3 efficiently prevents DEX-triggered mitochondrial dysfunction of myotubes through PGC1- $\alpha$ activities and its mitochondrial biogenetic transcription factors, NRF1 and Tfam. Rg3, ginsenoside Rg3; ROS, reactive oxygen species; DEX, dexamethasone; PGC1- $\alpha$, peroxisome proliferator-activated receptor $\gamma$ coactivator 1- $\alpha$; NRF1, nuclear respiratory factor 1; Tfam, mitochondrial transcription factor $\mathrm{A}$.

restored by $\mathrm{Rg} 3$ treatment (Fig. 3A). To further investigate, the differentiation of $\mathrm{C} 2 \mathrm{C} 12$ myoblasts was induced for three days in DM followed by the treatment with DEX with vehicle or $\mathrm{Rg} 3$ for additional one day (D4). Thereafter, the resultant cell lysates were subjected to immunoblotting for the expression of PGC1- $\alpha$, NRF1 and Tfam. The DEX treatment reduced the level of PGC1- $\alpha$, NRF1 and Tfam proteins, while Rg3 treatment partially restored the expression of these proteins in DEX-treated myotubes (Fig. 3B and C). Consistently with the results of immunoblotting results, the results of RT-qPCR analysis showed that $\mathrm{Rg} 3$ partially restored the transcription of PGC1- $\alpha$, NRF1 and Tfam in DEX-treated myotubes (Fig. 3D). In catabolic states, the loss of PGC1- $\alpha$ activates FoxO transcriptional factors to stimulate the atrophy process thereby promoting protein degradation (33). The overexpression of PGC1- $\alpha$ in muscle atrophy models has been reported to suppress FoxO3 and muscle-specific E3 ligases including MuRF1 and atrogin-1 thereby preventing muscle wasting and activate Akt/mTOR signaling thereby ameliorating cancer cachexia (33,34). Furthermore, the overexpression of PGC1- $\alpha$ in mouse skeletal muscles increases mitochondrial content and functions (35). Given these results, $\operatorname{Rg} 3$ upregulates the expression and activity of PGC1- $\alpha$ in DEX-treated myotubes, indicating that PGC1- $\alpha$ is a target of $\operatorname{Rg} 3$ leading to enhanced mitochondrial function.

\section{Rg3 improves mitochondrial functions in DEX-induced} myotubes. Mitochondrial dysfunction accompanied by generation of excessive reactive oxygen species leads to the elevation of oxidative stress associated with impaired skeletal muscle functions and the acceleration of the loss of muscle mass in a number of diseases including sarcopenia and cachexia (32). To examine the effect of $\mathrm{Rg} 3$ on mitochondrial ROS production in myotubes, the differentiation of $\mathrm{C} 2 \mathrm{C} 12$ myoblasts was induced in DM for three days and the resultant myotubes were treated with DEX together with vehicle or $\mathrm{Rg} 3$ for one day (D4) and subjected to mitochondrial ROS production analysis. As shown in Fig. 4A, mitochondrial ROS production significantly 
increased following the exposure of DEX in C2C12 myotubes, while being significantly inhibited by $\mathrm{Rg} 3$ treatment.

Decreased MMP reduces the capacity of mitochondrial ATP synthesis to meet cellular energy demands (36). Treatment with DEX reduces the MMP that was partially rescued by $\mathrm{Rg} 3$ treatment (Fig. 4B). In consistent with the result of MMP, DEX treatment led to lower ATP contents, compared with that of the control (Fig. 4C). However, Rg3 treatment restored ATP contents in DEX-treated myotubes, similarly to those in control myotubes (Fig. 4C). MMP is an index of the ability for ATP synthesis. Given these results, $\operatorname{Rg} 3$ protects myotubes from oxidative damage and mitochondrial dysfunction induced by DEX.

In conclusion, according to the findings of the present study, $\mathrm{Rg} 3$ activated Akt/mTOR signaling to prevent the atrophy of DEX-treated myotubes thereby suppressing the expression of muscle-specific E3 ubiquitin ligases, MuRF1 and atrogin-1 and protein degradation. Furthermore, Rg3 enhanced PGC1- $\alpha$ activities and its mitochondrial biogenetic transcription factors, NRF1 and Tfam thereby efficiently improved mitochondrial dysfunction triggered by DEX. Therefore, $\operatorname{Rg} 3$ is a potential therapeutic or nutraceutical remedy that protect muscles from muscle weakness and atrophy associated with chronic diseases and cancer.

\section{Acknowledgements}

Ginsenoside Rg3 was a gift from Dr Jeom-Yong Kim (Research Institute, Green Cross Wellbeing Co., Ltd., Seoul, South Korea).

\section{Funding}

The present study was supported by the National Research Foundation of Korea (NRF) grant funded by the Korea government (MSIP; grant no. NRF-2021-COMPA-0101 to GB; grant no. NRF-2017-R1D1A1B03032839 to SL).

\section{Availability of data and materials}

The datasets used and/or analyzed during the current study are available from the corresponding author on reasonable request.

\section{Authors' contributions}

SJL and GUB conceptualized the project and designed the experiments. RK, JWK and SJL performed the experiments. RK and SJL analyzed the results and performed the statistical analysis. SJL and GUB wrote the manuscript and supervised the project. SJL and GUB confirmed the authenticity of all the raw data. All authors have read and approved the final manuscript.

\section{Ethics approval and consent to participate}

Not applicable.

\section{Patient consent for publication}

Not applicable.

\section{Competing interests}

The authors declare that they have no competing interests.

\section{References}

1. Cohen S, Nathan JA and Goldberg AL: Muscle wasting in disease: Molecular mechanisms and promising therapies. Nat Rev Drug Discov 14: 58-74, 2015.

2. Argilés JM, Busquets S, Stemmler B and López-Soriano FJ: Cancer cachexia: Understanding the molecular basis. Nat Rev Cancer 14: 754-762, 2014.

3. Cao RY, Li J, Dai Q, Li Q and Yang J: Muscle atrophy: Present and future. Adv Exp Med Biol 1088: 605-624, 2018.

4. Singh A, Phogat J, Yadav A and Dabur R: The dependency of autophagy and ubiquitin proteasome system during skeletal muscle atrophy. Biophys Rev 13: 203-219, 2021.

5. Peris-Moreno D, Taillandier D and Polge C: MuRF1/TRIM63, master regulator of muscle mass. Int J Mol Sci 21: 6663, 2020.

6. Stefanetti RJ, Voisin S, Russell A and Lamon S: Recent advances in understanding the role of FOXO3. F1000Res 7: F1000, 2018.

7. Bae GU, Lee JR, Kim BG, Han JW, Leem YE, Lee HJ, Ho SM, Hahn MJ and Kang JS: Cdo interacts with APPL1 and activates Akt in myoblast differentiation. Mol Biol Cell 21: 2399-2411, 2010.

8. Kim JH: Cardiovascular diseases and panax ginseng: A review on molecular mechanisms and medical applications. J Ginseng Res 36: 16-26, 2012.

9. Chen XJ, Zhang XJ, Shui YM, Wan JB and Gao JL: Anticancer activities of protopanaxadiol- and protopanaxatriol-type ginsenosides and their metabolites. Evid Based Complement Alternat Med 2016: 5738694, 2016.

10. Kim MJ, Koo YD, Kim M, Lim S, Park YJ, Chung SS, Jang HC and Park KS: Rg3 improves mitochondrial function and the expression of key genes involved in mitochondrial biogenesis in C2C12 myotubes. Diabetes Metab J 40: 406-413, 2016.

11. Wang CZ, Aung HH, Zhang B, Sun S, Li XL, He H, Xie JT, $\mathrm{He}$ TC, Du W and Yuan CS. Chemopreventive effects of heat-processed Panax quinquefolius root on human breast cancer cells. Anticancer Res 28: 2545-2551, 2008.

12. Zhang L, Zhang L, Wang X and Si H: Anti-adipogenic effects and mechanisms of ginsenoside $\mathrm{Rg} 3$ in pre-adipocytes and obese mice. Front Pharmacol 8: 113, 2017.

13. Kim M, Ahn BY, Lee JS, Chung SS, Lim S, Park SG, Jung HS, Lee HK and Park KS: The ginsenoside Rg3 has a stimulatory effect on insulin signaling in L6 myotubes. Biochem Biophys Res Commun 389: 70-73, 2009.

14. Yoshida T and Delafontaine P: Mechanisms of IGF-1-mediated regulation of skeletal muscle hypertrophy and atrophy. Cells 9: 1970,2020

15. Lee SJ, Vuong TA, Go GY, Song YJ, Lee S, Lee SY, Kim SW, Lee J, Kim YK, Seo DW, et al: An isoflavone compound daidzein elicits myoblast differentiation and myotube growth. J Functional Foods 38: 438-446, 2017.

16. Lee SJ, Bae JH, Lee H, Lee H, Park J, Kang JS and Bae GU: Ginsenoside $\operatorname{Rg} 3$ upregulates myotube formation and mitochondrial function, thereby protecting myotube atrophy induced by tumor necrosis factor-alpha. J Ethnopharmacol 242: 112054, 2019.

17. Lee SJ, Im M, Park SK, Kim JY, So EY, Liang OD, Kang JS and Bae GU: BST204, a $\operatorname{Rg} 3$ and Rh2 enriched ginseng extract, upregulates myotube formation and mitochondrial function in TNF- $\alpha$-induced atrophic myotubes. Am J Chin Med 48: 631-650, 2020.

18. Lee SJ, Hwang J, Jeong HJ, Yoo M, Go GY, Lee JR, Leem YE, Park JW, Seo DW, Kim YK, et al: PKN2 and Cdo interact to activate AKT and promote myoblast differentiation. Cell Death Dis 7: e2431, 2016

19. Jeong HJ, Lee HJ, Vuong TA, Choi KS, Choi D, Koo SH, Cho SC, Cho H and Kang JS: Prmt7 deficiency causes reduced skeletal muscle oxidative metabolism and age-related obesity. Diabetes 65: 1868-1882, 2016.

20. Livak KJ and Schmittgen TD: Analysis of relative gene expression data using real-time quantitative PCR and the 2(-Delta Delta C(T)) method. Methods 25: 402-408, 2001.

21. Shanker G, Aschner JL, Syversen T and Aschner M: Free radical formation in cerebral cortical astrocytes in culture induced by methylmercury. Brain Res Mol Brain Res 128: 48-57, 2004. 
22. Murakami Y, Ueki R, Tachikawa T and Hirose M: The basic study of the mechanism of propofol-related infusion syndrome using a murine skeletal muscle injury model. Anesth Pain Med 9: e89417, 2019

23. Kim H, Cho SC, Jeong HJ, Lee HY, Jeong MH, Pyun JH, Ryu D, Kim M, Lee YS, Kim MS, et al: Indoprofen prevents muscle wasting in aged mice through activation of PDK1/AKT pathway. J Cachexia Sarcopenia Muscle 11: 1070-1088, 2020.

24. Martín AI, Priego T and López-Calderón A: Hormones and muscle atrophy. Adv Exp Med Biol 1088: 207-233, 2018.

25. Nakashima K, Ishida A, Ijiri D and Ohtsuka A: Effect of dexamethasone on the expression of atrogin-1/MAFbx in chick skeletal muscle. Anim Sci J 87: 405-410, 2016.

26. Baehr LM, Hughes DC, Lynch SA, Van Haver D, Maia TM Marshall AG, Radoshevich L, Impens F, Waddell DS and Bodine SC: Identification of the MuRF1 skeletal muscle ubiquitylome through quantitative proteomics. Function (Oxf) 2 zqab029, 2021.

27. Sakuma K, Aoi W and Yamaguchi A: Molecular mechanism of sarcopenia and cachexia: Recent research advances. Pflugers Arch 469: 573-591, 2017.

28. Sartori R, Romanello V and Sandri M: Mechanisms of muscle atrophy and hypertrophy: Implications in health and disease. Nat Commun 12: 330, 2021.

29. Glass DJ: PI3 kinase regulation of skeletal muscle hypertrophy and atrophy. Curr Top Microbiol Immunol 346: 267-278, 2010.

30. Go GY, Lee SJ, Jo A, Lee J, Seo DW, Kang JS, Kim SK, Kim SN, Kim YK and Bae GU: Ginsenoside Rg1 from Panax ginseng enhances myoblast differentiation and myotube growth J Ginseng Res 41: 608-614, 2017.
31. Go GY, Jo A, Seo DW, Kim WY, Kim YK, So EY, Chen Q, Kang JS, Bae GU and Lee SJ: Ginsenoside Rb1 and Rb2 upregulate Akt/mTOR signaling-mediated muscular hypertrophy and myoblast differentiation. J Ginseng Res 44: 435-441, 2020.

32. Yang S, Loro E, Wada S, Kim B, Tseng WJ, Li K, Khurana TS and Arany Z: Functional effects of muscle PGC-1alpha in aged animals. Skelet Muscle 10: 14, 2020.

33. Ruas JL, White JP, Rao RR, Kleiner S, Brannan KT, Harrison BC, Greene NP, Wu J, Estall JL, Irving BA, et al: A PGC-1a isoform induced by resistance training regulates skeletal muscle hypertrophy. Cell 151: 1319-1331, 2012.

34. Ascenzi F, Barberi L, Dobrowolny G, Villa Nova Bacurau A, Nicoletti C, Rizzuto E, Rosenthal N, Scicchitano BM and Musarò A: Effects of IGF-1 isoforms on muscle growth and sarcopenia. Aging Cell 18: e12954, 2019.

35. Gao X, Zhao XL, Zhu YH, Li XM, Xu Q, Lin HD and Wang MW: Tetramethylpyrazine protects palmitate-induced oxidative damage and mitochondrial dysfunction in $\mathrm{C} 2 \mathrm{C} 12$ myotubes. Life Sci 88: 803-809, 2011

36. Yan XH, Guo XY, Jiao FY, Liu X and Liu Y: Activation of large-conductance $\mathrm{Ca}(2+)$-activated $\mathrm{K}(+)$ channels inhibits glutamate-induced oxidative stress through attenuating ER stress and mitochondrial dysfunction. Neurochem Int 90: 28-35, 2015.

This work is licensed under a Creative Commons Attribution-NonCommercial-NoDerivatives 4.0 International (CC BY-NC-ND 4.0) License. 